\title{
Risk Trends, Indicators and Learning Rates: A New Case Study of North Sea Oil and Gas
}

\author{
R.B. Duffey \\ Atomic Energy of Canada Limited, Chalk River, Ontario K0J 1J0 Canada, e-mail: duffeyr@aecl.ca
}

A.B. Skjerve

Institute for Energy Technology, P.O. Box 173, NO-1751 Halden, Norway, Email:ann.britt.skjerve@hrp.no

\begin{abstract}
Industrial accidents, explosions and fires have a depressingly familiar habit of re-occurring, with similar if not identical causes. There is a continual stream of major losses that commonly are ascribed to poor operating and management practices. The safety risks associated with modern technological enterprises make it pertinent to consciously monitor the risk level. A comprehensive approach in this respect is being taken by the Petroleum Safety Authority Norway (PSA)'s program "Trends in Risk Levels Norwegian Continental Shelf." We analyse the publicly available data provided by this program using the Duffey-Saull Method. The purpose of the analysis is to discern the learning trends, and to determine the learning rates for construction, maintenance, operation and administrative activities in the North Sea oil and gas industry. This outcome of this analysis allows risk predictions, and workers, management and safety authorities to focus on the most meaningful trends and high-risk activities.
\end{abstract}

\section{INTRODUCTION}

The offshore oil and gas industry is a huge and modern technological enterprise in which vast quantities of oil are pumped, shipped, and stored. These large socio-technological facilities pose major hazards, with potential for spills, fires, sinkings and explosions in a hazardous and sensitive sea environment with risk of accident, injury and death to the operators. In his review of major oil industry failures and engineering practices (Moan 2004) assesses that: "the main cause of actual structural failures are due to human errors and omissions ... and cause $80-90 \%$ of the failures of buildings, bridges and other engineering structures." This is also true with what is found in all other industries and technological systems world wide, and the same types of mistakes and common errors appear (Duffey \& Saull 2002). Industrial accidents, explosions and fires have a depressingly familiar habit of re-occurring, with similar if not identical causes. There is a continual stream of major losses that commonly are ascribed to poor operating and management practices, as in recent examples of sometimes billion dollar damage and losses at offshore oil rigs (e.g. the P-36 platform that sank following three explosions on March 2001), oil storage facilities (e.g. the Buncefield Oil Depot explosions and fire of December 2005), and refineries (e.g. the catastrophic process accident on the BP Texas City refinery of March 2005). This has extreme significance in risk management, and great importance in the development of safety manage- ment systems, not to mention the large impact on insurance costs, corporate liability and losses, and the threat to worker safety. This is important not only for such large disasters but also for everyday accidents, where the issue is: How can a large facility loss be predicted using the everyday events and operation of that facility.

The safety risks associated with modern technological enterprises make it pertinent to consciously monitor the risk level, to assess the extent to which safety improving initiatives are required. A comprehensive approach in this respect is being taken by the Norwegian oil and gas regulator, the Petroleum Safety Authority Norway (PSA), who are responsible for overseeing the safety of many of the massive deep sea platforms operating in the storm swept North Sea. The PSA's newly developed program called on "Trends in Risk Levels Norwegian Continental Shelf", where key measures have been defined for the purpose of tracking and evaluating both relative safety improvements in specific areas plus defining an overall risk level, with the objective "to create a reliable decision making platform for industry and authorities" (PSA 2003). In a wide-range approach to "measure risk for an entire industrial sector", twenty-one (21) risk indicators define situations of hazard and accident (called DFUs), covering many known major and minor outcomes. These indicators include data for the differing activity segments of the oil and gas offshore and onshore work (shipping, transport, maintenance ...) and in- 
clude events and abnormal activities (leaks, accidents and incidents ...), plus the effectiveness of "barriers" (systems, practices and procedures ...). The yearly trends of the quantitative data are analyzed as to whether these show change (increase, decrease, or not) of both the numbers and rates of indicator outcomes; and whether there is any relation to more qualitative measures based on attitudinal surveys.

Determining the safety level based on this type of calculation can, however, be a difficult task. The recent PSA report states: "On the basis of the data and indicators used in this project, no clear positive or negative trends can be observed in risk level. Most major accident indicators show an improvement in 2003 in relation to 2002. Serious injuries to personnel also show a decrease in 2003. The position is now on a level with the average for the previous 10 years. Cooperation and trust between the parties are seen as good."

Since the common factor and major cause in industrial accidents everywhere is the human involvement, it is postulated here that by understanding the prior outcomes, human learning and error correction, we can predict the probability of observing any outcome. The key questions to answer when looking at trends are: Are we learning from our past mistakes? What is the rate of learning now? What is it predicted to be in the future?

Precisely to quantify such issues, Duffey \& Saull (2002) have derived measures and methods for the analysis of learning rates as direct indicators of safety improvement using existing worldwide outcome data for some 200 years and covering over 60 examples. The approach, called the Duffey-Saull Method (DSM), uses the Learning Hypothesis to analyze and predict errors, accidents, injuries and all other such risk outcomes as a function of experience. It assumes that with continuous exposure to a given operational setting humans will learn to master task performance, and that the manifest effect of learning will be lower accident/incident rates - because humans as a starting point is assumed to be the key contributing factor to accident/incidents. The present Case Study applies these techniques and approaches to analyze the new and publicly available North Sea outcome data. Using the experiencebased DSM, we try to discern the learning trends, and determine the learning rates for construction, maintenance, operation and drilling activities in the North Sea oil and gas industry. In our Case Study, we provide a basis to determine, prioritize, and compare the learning rates and injury trends between different key work phases. This analysis allows risk predictions, and provides guidance for workers, management and safety authorities to focus on the most meaningful trends and high-risk activities.

\section{RISK INDICATOR DATA ANALYSIS}

The procedure we use is to first determine the risk outcomes, rates and numbers, and their distribution with experience. The basic prior data for Norway for 1996-2005 are reported by the PSA in both graphical and tabular form (PSA 2007). Some equivalent data for the UK for 1992-2002 are tabulated in Yang \& Trbojevic 2007 (Table 6.16 p 195). All the data are typically given and are analyzed by calendar year, such as the number of injuries to workers, broken down by different sub-categories of severity (e.g., major or total), and work location and/or activity type (e.g., fixed or mobile facility, drilling or maintenance).

To convert to a learning basis for analysis, we use the relevant measure of experience as the accumulated worker-hours, summing the year-by-year numbers reported. A typical xls. spreadsheet tabulation and analysis of the rates is shown in Table 1 for a subset in our observational interval. In this case, the data are for injuries in drilling at fixed facilities for Norway, and similar tables were made for all the various sets where numbers were available.

Table 1. Typical data subset - Norway well drilling injuries 1996-2005

\begin{tabular}{crrrrrr}
\hline $\begin{array}{c}\text { Well drilling } \\
\text { (hours) }\end{array}$ & $\begin{array}{c}\text { Injuries, } \\
\mathbf{n}\end{array}$ & \multicolumn{1}{c}{ AccMh } & \multicolumn{1}{c}{$\mathbf{N}^{*}$} & \multicolumn{1}{c}{$\begin{array}{c}\text { Entropy, } \\
\text { Injury } \\
\text { Rate/Mh }\end{array}$} & Year \\
\hline 4670117 & 145 & 4.670117 & 0.088633 & 0.27047 & 31.04847 & 1996 \\
4913477 & 141 & 9.583595 & 0.181884 & 0.266685 & 28.69658 & 1997 \\
4967799 & 133 & 14.551394 & 0.276167 & 0.258794 & 26.77242 & 1998 \\
4418068 & 117 & 18.969462 & 0.360016 & 0.241637 & 26.48216 & 1999 \\
4696224 & 121 & 23.665686 & 0.449144 & 0.246107 & 25.76538 & 2000 \\
5168486 & 110 & 28.834172 & 0.547236 & 0.233505 & 21.28283 & 2001 \\
5506589 & 103 & 34.340761 & 0.651744 & 0.224957 & 18.70486 & 2002 \\
5827360 & 90 & 40.168122 & 0.762339 & 0.207881 & 15.44438 & 2003 \\
6248973 & 54 & 46.417095 & 0.880937 & 0.150437 & 8.64142 & 2004 \\
6273504 & 59 & 52.690599 & 1 & 0.159497 & 9.404633 & 2005 \\
\hline
\end{tabular}

This Table is in general for the $\mathrm{j}^{\text {th }}$ observation interval, with the sub-intervals within it. Such a tabulation is not by itself very informative, apart from illustrating the manipulations and steps in the necessary arithmetic for each experience increment:

1 adding up prior worker-hours to obtain the running total of the accumulated millions of hours of experience, $\varepsilon$ (AccMh) for each $i^{\text {th }}$ sub-interval;

2 turning the injury numbers, $n_{i}$, into risk Rates per Mh by straightforward division;

3 calculating the non-dimensional experience, $\mathrm{N}^{*}$, by dividing each AccMh interval, $\varepsilon_{i}$, by the total accumulated experience, $\varepsilon_{\mathrm{T}}\left(\varepsilon_{\mathrm{T}}=\Sigma \mathrm{AccMh}=\right.$ $53 \mathrm{Mh})$; and

4 calculating the entropy $\left(\mathrm{H}_{\mathrm{i}}=\mathrm{p}_{\mathrm{i}} \ln \mathrm{p}_{\mathrm{j}}\right)$ in each $\mathrm{i}^{\text {th }}$ sub-interval from the probability, where $\mathrm{p}_{\mathrm{i}}=$ $\mathrm{n}_{\mathrm{i}} / \mathrm{N}_{\mathrm{j}}$, where, $\mathrm{N}_{\mathrm{j}}$, is the total number of injuries $\left(\mathrm{N}_{\mathrm{j}}\right.$ $=\Sigma \mathrm{n}_{\mathrm{i}}=1073$ ).

To clarify the trends, typical results of such analysis of the raw data are then plotted in Figure 1. The figure also shows some UK data alongside the Norway data. By grouping the data together in this 
way, several key comparisons and observations are possible. In addition, to simplify the presentation, simple exponential fits are shown to the data, since we expect such a curve to crudely represent the improvement effects of learning (Duffey \& Saull 2002).

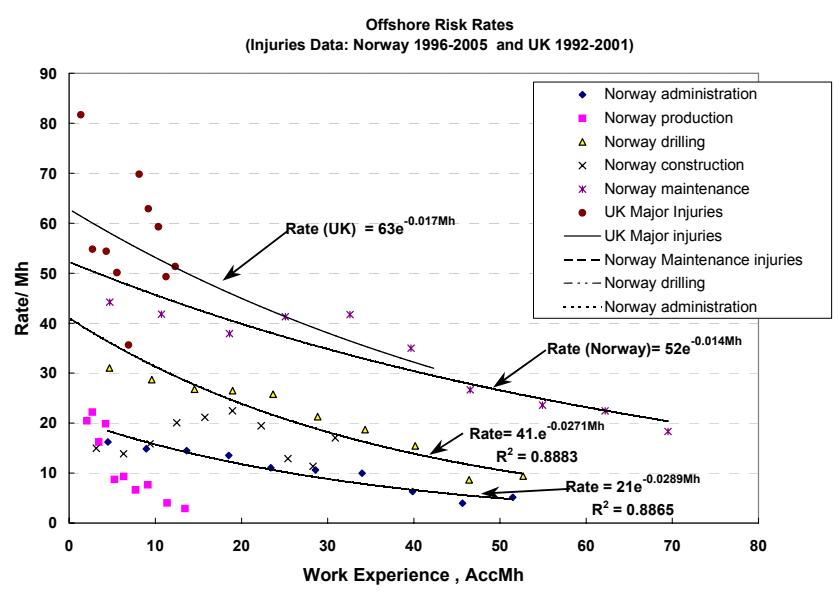

Figure 1. Typical data plot and simplified curve fits.

Firstly, learning is evident in most of the data, but the absolute risk indicator rates are higher for some activities, and some task areas are clearly learning slower than others (the slope is half). We may predict that they will all reach some asymptotic but slow learning state, by about twice the present experience if learning continues. The most hazardous (highest risk) activities are clearly maintenance and drilling, and must be the areas of most safety importance and management attention.

Secondly, the lowest rates attained so far (in administration and production) are $\sim 5 / \mathrm{Mh}$, or about 1 in 200,000 experience hours, in complete accord with the lowest risk found in any other industry (Duffey \& Saull 2002). However, the highest rates are ten times more, or $\sim 1$ in 20,000 experience hours, which is also comparable to other industries.

Thirdly, the UK has less experience, but a simple extrapolation forward of the rough fit to the major injury (MI) data, and backward extrapolation of the Norway maintenance data shows similar event rate magnitudes and learning rates. The implication is that learning of similar effectiveness in Norway and the UK suggests that further research is needed into the influencing factors and causes of this similarity. Thus, we may predict and expect the UK rates to fall further and track down towards the Norway risk rates if such international learning continues. Similar convergence trends are observed with differing experience in, say, commercial aircraft near-misses and marine shipping accidents.

\section{ARE WE DOING ENOUGH TO ENSURE}

\section{SAFETY?}

A key question for managers of hazardous industries is: Are we doing enough to ensure safety? In this section we will take a closer look at learning in a workplace setting, and suggest that this question may also be answered based on an assessment of the effectiveness of the joint initiatives taken by an organization (or an entire industry) to ensure safety.

Individuals learn as they gain experience (Ebbinghause 1885). Employees in petroleum companies will learn from participation in the formal education and training programs offered by their organization. The aim of these programs is to ensure that all employees possess the competence, i.e. the skills, knowledge and attitudes required to efficiently perform their jobs to the specified standard (IAEA 2002; Skjerve \& Torgersen 2007). As part of their engagement in the every-day work activities, the employees will moreover face a range of learning opportunities resulting from the myriad of different situations that arise from interactions between humans, technology and administrative systems. The employees will need both the competence acquired from the formal education/training sessions and the competence acquired based on the more informal experiences gained on-the-job, to be able to perform their tasks efficiently (Johnston \& Hawke 2002). With increased experience, employees will obtain still more refined insights into the task performance process and their task performance environment, ${ }^{1}$ and gradually they will be able to perform the routine part of their tasks in a highly automated manner (Rasmussen 1986).

Observation, imitation, reflection, discussion, and repetition may all constitute important elements in employees' learning processes. Handling of situations where unexpected occurrences happen in relation to task performance provides an important basis for learning. Such unexpected occurrences may be caused by human errors (e.g. errors of the particular employee, errors of colleagues - in some situations the errors may even be consciously introduced for the employees to learn something). Unexpected occurrences may also be caused by breakdowns in technology or administrative systems, or by any combination of the above factors. When unexpected occurrences arise things will not progress according to plan, and this will spur the employees to develop a more comprehensive understanding of the task performance process and the work environment. This, in turn, will improve their ability to perform safely in future situations. Accidents constitute important, but highly unwarranted, learning opportunities. When accidents happen, they will tend to chal-

\footnotetext{
${ }^{1}$ The extent to which this process involves deduction based on inference rules or the development of mental models is still a matter of debate (cf., e.g. Johnson-Laird \& Byrne 2000).
} 
lenge the organization's model of the risks it faces and the effectiveness of its countermeasure (Woods 2006). For this reason, radical changes may be implemented in the organization following an accident investigation. This suggests that not only individuals but also the organization as such may learn from experience.

Organizational learning may be defined as “... the capacity or processes within an organization to maintain or improve the performance based on experience" (DiBella 2001, Duffey \& Saull 2002). A key element in organizational learning is the transformation of experiences gained by employees to the organizational level. In this process, however, the organization needs to be aware that not all the experiences gained by employees will contribute to increase the likelihood for safe performance: Employees are engaged in a continuous learning process. Misunderstandings of factors in the work environment, misunderstandings of the inter-relationship between these factors, inaccurate risk perception, etc. can all be expected to be (intermediate) elements or states in a learning process. In addition, to the experiences of the employees, experiences obtained by other organizations or by other industries may also prove valuable to organizational learning. Organizational learning should be manifest in the structures and processes of the organization (Svenson 2006). Concretely, organizational learning may result in the introduction of new work practices, revisions of operational procedures, refinements of training programs, improvement in the safety management approach, etc. That is, in initiatives that jointly aim at ensuring safe and efficient production.

To facilitate learning processes at all levels in the organization it is important to ensure that a learning culture is engineered (Reason 1997). A learning culture can be defined as "... an environment in which opportunities for learning are openly valued and supported and are built, where possible, into all activities" (DEST 2005). It has been suggested that effective high-reliability organizations is characterised by their ability to learn as much as possible for the failures that occur (Weick \& Sutcliffe 2001).

Finally, the importance of ensuring a sound safety culture is generally reckoned as a prerequisite for safe production in high-risk industries. The agenda among most actors in the Norwegian petroleum sector is to improve the safety culture both within and across the industry (Hoholm 2003). Safety culture can be defined as "...that assembly of characteristics and attitudes in organizations and individuals which establishes that, as an overriding priority, safety issues receive the attention warranted by their significance." (Adapted from IAEA 1991, Yang \& Trbojevic 2007). A sound safety culture means that the structures and process of the organization should work together to ensure safety. Thus, deviations caused by the activities in one part of the organization should be compensated by the activity in other parts of the organization so that safety is always ensured (Weick \& Sutcliffe 2001). A sound safety culture, moreover, implies that the attitudes and behaviours of employees should promote safety. In the context of the Norwegian petroleum industry, the impact of colleagues' and managers' attitudes to safety on the individual employee at was demonstrated in two recent studies (Aase et al. 2005; Skjerve, in press).

One way to answer the question: "Are we doing enough to ensure safety?" could be to calculate the effectiveness of the joint initiatives taken by an organization (or an entire industry) to ensure safety. In the next section, we introduce " $H$ " as one such possible measure.

\section{SAFETY CULTURE, RISK MANAGEMENT AND PREDICTION}

The emergence of order from chaos is a precise analogy to that postulated for microscopic physical and chemical systems (Prigogine 1984), and is the expressed intent of safety management and risk indicators for macroscopic socio-technological systems. In particular, we determine the Information Entropy risk measure, H, which Duffey \& Saull (2007) suggest is the objective and quantitative measure of safety culture, management systems, organizational learning and risk perception. Thus, since we may regard, $\mathrm{H}$, as a measure of the "disorder" this, of course, is the converse of "order", and hence is an indication of the effectiveness of these safety management processes.

The statistical theory that determines the outcome risk distribution yields an explicit expression for the Information Entropy, H, using the probability of the outcomes (Pierce 1980, Jaynes 2003, Duffey \& Saull 2004, Duffey \& Saull 2008). The degree of order is a function of the depth of experience based on the frequency of error state occupation, $n_{i}={ }_{i} N_{j}$.

The classic result for the Information Entropy, H, is a measure of the uncertainty, or the "missing information" or the "degree of order" given by:

$$
\mathrm{H}_{\mathrm{j}}=-\Sigma \mathrm{p}_{\mathrm{i}} \ln \mathrm{p}_{\mathrm{i}}
$$

Substituting in the expression for the Information Entropy, H, in the companion paper (Duffey \& Saull 2008), we obtain:

$$
\mathrm{H}_{\mathrm{j}}=1 / 2\left\{\mathrm{p}_{0} \mathrm{e}^{-\mathrm{aN} *}\right\}^{2}\left\{\mathrm{aN}^{*}+1 / 2\right)
$$

The relative value of the information entropy, $\mathrm{H}$, at any experience depth is also an objective measure of the cultural aspect of modern technologies called "organizational learning" since it reflects the degree of learning and the extent of management "order" or 
effectiveness. The so-called organizational learning and safety culture attributes of a HTS, and its management's ability to respond effectively to the demands for continuous safety improvement. The resulting structure and probability of observed outcomes are a direct reflection of the internal organizational and skill acquisition caused by the innumerable human learning and unlearning interactions occurring within.

These statistical fluctuations, due to human decision making and actions, also cause the uncertainty of precisely when an event will actually occur, which uncertainty is determined and measured by the information entropy. The unobserved random fluctuations produced by unpredictable human behavior (the chaos) are reflected in the emergent order at the system level: namely, the observed predictable trend of learning and error reduction with increasing experience. Thus the influence of safety management can be quantified, and predictions made about the effectiveness of learning and management systems. By providing the information entropy, H-measure (which we can also refer to as the "Learning Entropy"), we can not only describe but also can predict the impact of the learning behavior on the degree of order and on the risk trends with experience that are attained.

\section{COMPARISON OF THEORY AND DATA}

For the present Case Study, we can now compare this theory to the overall trends of a subset of the present risk indicator data, noting that we have evaluated the entropy already as part of the initial data analysis (see Table 1). To simplify, the data are normalized to the initial probability at the initial or lowest experience, where we take, $\mathrm{p}_{0}=1$, by definition. Figure 2 shows the Norway (injury) and UK (major injury and $>3$ day injury) data compared to the theory (SEST) prediction, but adopting a value of, $a=1$, for the shape or slope parameter in the entropy distribution.

Rather satisfyingly, the theory and data easily appear side-by-side on the same graph, lending some credence to this analysis. The other data shown for comparison purposes are the commercial aircraft near-misses (NMACs), because of the significant and traditional airline emphasis on safety (Duffey \& Saull 2002). The NMAC line up rather better with the theoretical prediction, but there are clearly some key differences between the oil and gas data set trends. Despite the scatter, we note for this data subset that the:

1 entropy distribution with experience lies above the theory line;
2 slope trend is less than the theory, indicating insufficient attainment of order;

3 data lie above the best aircraft practices (aircraft near-misses); and

4 best (but still far from perfect) fit to all the injury data is a straight line, not an exponential as we should expect.

The approximate straight line "fit" shown is, $\mathrm{H}=$ $0.28-1.11 \mathrm{~N}^{*}$, which actually corresponds to the first two terms of the series expansion of the rather slowly-decaying exponential. Therefore, the implied first-order approximate value is $\mathrm{a} \approx 1.11$ for the distribution exponent.

All these trends and comparisons suggest symptoms of potentially insufficient learning, giving inadequate reduction in risk compared both to the expected ideal, and other industries. This adverse trend was confirmed by plotting the rates against the Universal Learning Curve, and finding a similar value of, $\mathrm{k} \sim 1$, for the learning rate constant.
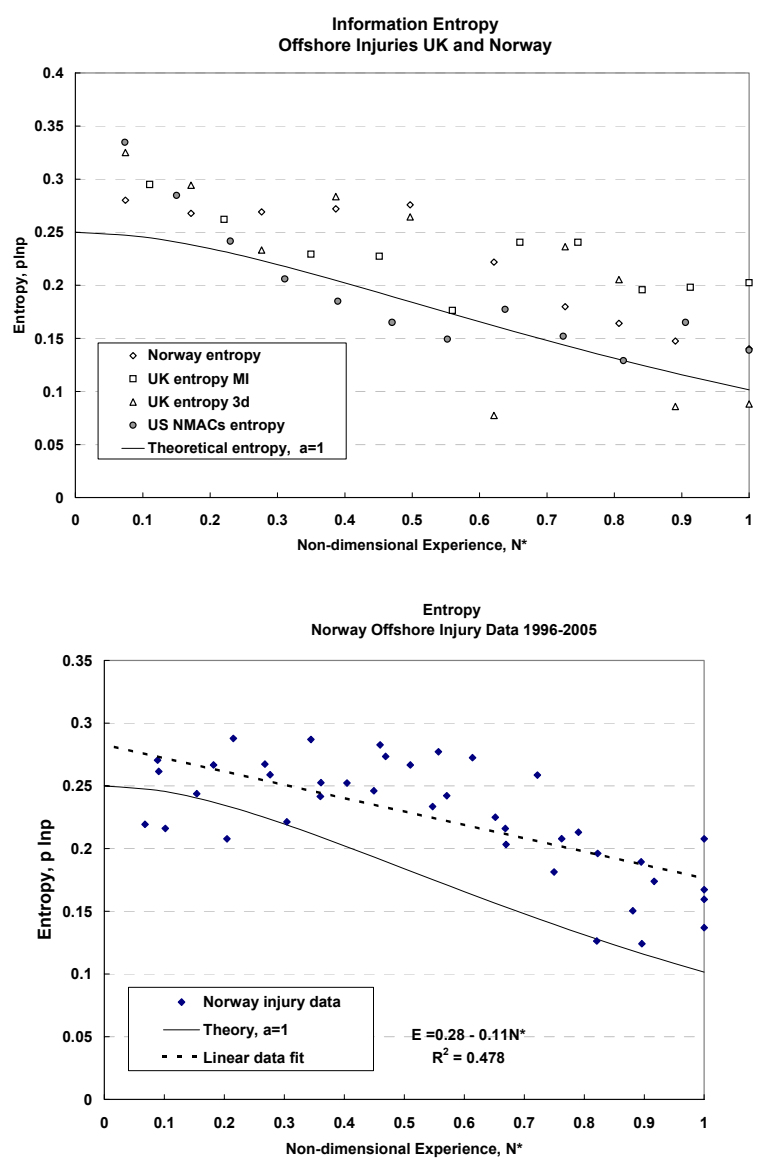

Figure 2. Comparisons of Theory and Data.

\section{CONCLUSIONS AND OBSERVATIONS}

We are interested in predicting safety performance and accident occurrences utilizing quantitative analysis of prior data. These predictions should serve to inform the industry to facilitate decision making with respect to when more emphasis on safety initiatives is required. As the predictions ex- 
press learning rates, they will allow companies to readily compare their learning rate with other companies in the same domain to establish whether they are on the right track. Likewise, the predictions allow for comparisons between entire industrial sectors, and they may in this way contribute to decisions of the national safety authorities when defining requirements to the various industrial sectors.

The two types of representations presented in this paper in Figure 1 and Figure 2 invites different interpretations of what spurs the learning rate. The representation used in Figure 1 may invite the interpretation that further accidents/incidents are necessary for learning to take place whereas Figure 2 suggests that we can intercompare learning and progress using the existing knowledge.

Still, even if handling of unexpected events is a key element in the learning process (as discussed above), this does not imply that accidents/incidents will have to take place for people to learn. Accidents/incidents will only occur when the organization is not sufficiently robust to prevent that human performance will have adverse implications. The fact that the learning rate seems to decrease only when incidents and accidents occur is caused by the fact that accidents/incidents (rather than e.g. successful outcomes) serves as input data for the model. In general accidents/incidents can be expected to occur in lower frequencies as an organization or an entire industrial section gains experience. This point of view is emphasised by the representation contained in Figure 2. It shows the learning rate based on the level of control an organisation or and industrial section have over the production processes.

Based on our Case Study of the observed and published safety indicator data for some ten years of operation of Norway and UK North Sea oil and gas facilities, and the trends shown in a subset of the data, we observe that:

- Learning is occurring in the major risk indicators as experience is gained, and this trend is similar between the Norway and UK indicating some commonality in approach and safety standards (after correction for differing experience);

- Certain activities, notably maintenance and drilling, apparently have much higher risk than others, both in numbers and rates, and suggest themselves as priority areas for management emphasis; and

- Evaluation of the Learning Entropy as a measure of the degree of order attained by safety management (suggested to represent organizational learning and safety culture) also indicate symptoms of potentially insufficient learning.

Extension of this Case Study to the complete set of risk indicators would be desirable; as also would revising the indicator choices to reflect priority of risk-related importance; and changing the conven- tional purely time-series manner of data reporting and analysis.

\section{REFERENCES}

Aase, K., Skjerve, A.B.M. \& Rosness, R. 2005. Why Good Luck has a Reason: Mindful Practices in Offshore Oil and Gas Drilling. In: S. Gherardi \& D. Nicolini (eds.), The Passion for Learning and Knowing. Proceedings of the 6th International Conference on Organizational Learning and Knowledge, vol. 1.: 193-210. Trento: University of Trento e-books.

DEST, 2005. The website of the Department of Education, Science and Training of Australia. http://www.dest.gov.au/sectors/training_skills/policy is-

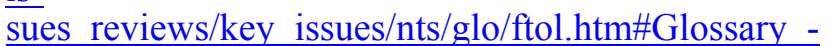
L (Accessed January 2008)

DiBella, A.K. 2001. Learning practices: Assessment and Action for Organizational Improvement. Upper Saddle River, N.J: Prentice-Hall.

Duffey, R.B. \& Saull, J.W. 2002. Know the Risk, First Edition, Boston, USA, Butterworth and Heinemann.

Duffey, R.B. and Saull J.W. 2004. Reliability and Failures of Engineering Systems Due to Human Errors, Proc. The First Cappadocia Int. Mechanical Engineering Symposium (CMES'-04), Cappadocia, Turkey.

Duffey, R.B. and Saull, J.W. 2007. Risk Perception in Society: Quantification and Management for Modern Technologies, Proc. Safety and Reliability Conference, Risk Reliability \& Societal Safety (ESREL 2007), Stavanger, Norway, 24-27 June.

Duffey, R.B. and Saull, J.W. 2008. Risk Management Measurement Methodology: Practical Procedures and Approaches for Risk Assessment and Prediction, Proc. ESREL 2008 and $17^{\text {th }}$ SRA Europe Annual Conference, Valencia, Spain, 22-25 September.

Ebbinghaus, H. 1885. Memory: A Contribution to Experimental Psychology. (Translated from: "Über das Gedächtnis"). http://psy.ed.asu.edu/ classics/Ebbinghaus/index.htm (Accessed January 2008).

Hoholm, T. 2003. Safety Culture in the Norwegian Petroleum Industry: Towards an Understanding of interorganisational culture development as network learning. Arbeidsnotat nr. 23/2003. Oslo: Center for Technology, Innovation and Culture, University of Oslo.

IAEA, 1991. Safety Culture, Safety Series no. 75-INSAG-4, Vienna: International Atomic Energy Agency.

IAEA, 2002. Recruitment, Qualification and Training of Personnel for Nuclear Power Plants, Safety Guide no. NS-G2.8, Vienna: International Atomic Energy Agency.

Jaynes, E.T. 2003. Probability Theory: The Logic of Science, First Edition, Edited by G.L. Bretthorst, Cambridge University Press, Cambridge, $U K$.

Johnston, R. \& Hawke, G. 2002. Case studies of organisations with established learning cultures, The National Centre for Vocational Education Research (NCVER), Adelaide, Australia. http://www.ncver.edu.au/research/proj/nr9014.pdf (Accessed January 2008)

Johnson-Laird, P. \& Byrne, R., 2000. Mental Models Website. http://www.tcd.ie/Psychology/Ruth Byrne/mental m odels/ (Accessed January 2008)

Moan, T. 2004. Safety of Offshore Structures, Second Keppel Offshore and Marine Lecture, CORE Report No. 2005-04, National University of Singapore. 
Petroleum Safety Authority Norway (PSA) 2003. Trends in risk levels-Norwegian Continental Shelf, Summary Report, Phase 4-2003, Ptil-04-04, p.11, Norway.

Petroleum Safety Authority Norway (PSA) 2007. Supervision and Facts, Annual Report 2006, Stavanger, Norway, 26 April, available at www.ptil.no)

Pierce, J.R. 1980. An Introduction to Information Theory, Dover, New York.

Prigogine, I. and Stengers, I. 1984. Order Out of Chaos: Man's New Dialogue with Nature, Toronto, Bantam Books.

Rasmussen, J. 1986, Information Processing and HumanMachine Interaction. An Approach to Cognitive Engineering, System Science and Engineering, vol. 12, New York: North-Holland.

Reason, J. 1997. Managing the Risks of Organizational Accidents. Aldershot, UK: Ashgate.

Skjerve, A.B. (in press). The Use of Mindful Safety Practices at Norwegian Petroleum Installations. To be published in Safety Science.

Skjerve, A.B. \& Torgersen, G.E. 2007. An OrganizationalPedagogical Framework to Support Competence Assurance Activities. In: T. Aven, J.E.Vinnem (Eds.), Risk, Reliability and Societal Safety: 1925-1932. London, UK: Taylor \& Francis Group,

Svenson, O., 2006. A Frame of Reference for Studies of Safety Management. In (Ed.) O. Svenson, I. Salo, P. Oedewald, T. Reiman, A.B. Skjerve, Nordic Perspectives on Safety Management in High Reliability Organizations. Theory and Applications: 1-7. Valdemarsvik, Sweden: Stockholm University.

Weick, K.E. \& Sutcliffe, K.M. 2001. Managing the Unexpected. Assuring High Performance in an Age of Complexity. San Francisco, CA: Jossey Bass.

Woods, D.D. 2006. Essential Characteristics of Resilience. In E. Hollnagel, D.D. Woods \& N. Leveson, Resilience Engineering. Concepts and Precepts:21-34. Aldershot, UK: Asgate.

Yang, J. \& Trbojevic, V. 2007. Design for Safety of Marine and Offshore Systems, IMarEST Publications, ISBN: 1902536-58-4. 